\title{
An average formula for the class number
}

\author{
by \\ Fernando Chamizo and Adrián Ubis (Madrid)
}

1. Introduction. In Art. 302 and Art. 304 of Disquisitiones Arithmeticae, Gauss considered averages of the class number of primitive integral binary quadratic forms. In the first of them, he dealt with the case of negative discriminants and claimed that he had found "by a theoretical investigation" a mean value formula that in modern notation can be written as

$$
\sum_{n \leq N} h(-4 n) \sim \frac{4 \pi}{21 \zeta(3)} N^{3 / 2}-\frac{2}{\pi^{2}} N .
$$

Most probably, Gauss proved this asymptotic formula using the lattice point interpretation of class number ([Ga, Art. 172, Art. 174]). It seems that the first published proof is due to Lipschitz [Li] and the best known bound for the error term (see the note at the end of [Ch-Iw2]) is

$$
\sum_{n \leq N} h(-4 n) \sim \frac{4 \pi}{21 \zeta(3)} N^{3 / 2}-\frac{2}{\pi^{2}} N+O\left(N^{\alpha}\right) \quad \text { for every } \alpha>\frac{21}{32} .
$$

The case of positive discriminants has remained mysterious up to the present day (see [Sa1] and [Sa2] for astonishing average formulas over special sequences). Gauss mentioned in Art. 304 that the product of the class number by the logarithm of the fundamental unit seems to behave in a regular way (this is natural by the class number formula). In fact, more than a century later, Siegel [Si] proved

$$
\sum_{n \leq N} h(4 n) \log \varepsilon_{4 n}=\frac{4 \pi^{2}}{21 \zeta(3)} N^{3 / 2}+O(N \log N) .
$$

(Here $\varepsilon_{4 n}$ is the "fundamental unit" as explained below.)

It is interesting to recall that originally Art. 304 of Disquisitiones Arithmeticae [Ga] reads: "[...] the average value of that product is approximately

2000 Mathematics Subject Classification: 11E41, 11L07, 11P21.

Research partially supported by the grant MTM 2005-04730 of the MEC. The second author is also supported by an FPU grant of MEC. 
expressed by a formula like $m \sqrt{D}-n$. However, we have not thus far been able to determine the values of the constant quantities $m, n$ theoretically. If it is permissible to draw a conclusion from the comparison of some hundreds of determinants, $m$ seems to be very nearly $7 / 3$ ". This is not correct because the value of $m$ corresponds to $3 / 2$ of the coefficient in Siegel approximation (Gauss looked for $m$ and $n$ such that $\sum(m \sqrt{D}-n) \sim \sum h(4 D) \log \varepsilon_{4 D}$, see Corollary 1.3), hence the actual value of $m$ is $2 \pi^{2} /(7 \zeta(3))=2.345 \ldots$, so $m-7 / 3 \approx 0.01$. On the other hand, the work of Shintani [Sh] shows that even the form of Gauss approximation is wrong because $n$ should be replaced by $n^{\prime} \log D$. It is impressive that Gauss could extend his calculations to several hundreds, but it is not strange that in this range he could not distinguish a logarithm from a constant in an approximated formula.

The right value of $m$ was given by Gauss in one of his handwritten notes (see [Ga, p. 462]). That note suggests he had a proof which "illustrates brilliantly many parts of higher Arithmetic and Analysis"; it is likely that he referred to a lattice point argument (cf. [Si]).

In this paper we are going to sharpen the results of Shintani [Sh], getting in the analogue of (2) an error term as in (1). We have to face the same problem as Gauss probably found when he guessed his formula for positive discriminants: there is no neat interpretation of $h(n)$ or $h(n) \log \varepsilon_{n}$ as a lattice point count when $n>0$. This obstructs a direct approach. Besides that, "Poisson summation" is technically more complicated in the positive discriminants case (compare Propositions 2.3 and 2.4 with Lemma 3.1 of [Ch-Iw2]).

Before stating our main results we make some remarks about notation. As usual, we denote by $h(n)$ the class number of primitive quadratic forms of discriminant $n$. We average over all discriminants (not only fundamental ones, for that case see [Go-Ho]), hence $n$ is an arbitrary positive integer. Of course, if $n \not \equiv 0,1(\bmod 4)$ we have $h(n)=0$. If $n$ is not a square, the "fundamental unit" $\varepsilon_{n}$ is $(t+u \sqrt{n}) / 2$ with $t, u \in \mathbb{Z}^{+}$the smallest solution of the Pell equation $t^{2}-n u^{2}=4$. If $n$ is a square this equation has $(t, u)=(2,0)$ as a unique non-negative solution and we write $\varepsilon_{n}=1$, then $\log \varepsilon_{k^{2}}=0$ and there is no contribution from square discriminants.

Gauss only considered quadratic forms $a x^{2}+b x y+c y^{2}$ with $b$ even, which implies that his definition of class number for discriminant $n$ coincides with $h(4 n)$ in modern notation. With the current definition this factor 4 is unnatural and we omit it in our first main result.

Theorem 1.1. For every $\alpha>21 / 32$,

$$
\sum_{n \leq N} h(n) \log \varepsilon_{n}=\frac{\pi^{2}}{18 \zeta(3)} N^{3 / 2}-\frac{3}{\pi^{2}}(C+\log N) N+O\left(N^{\alpha}\right)
$$

where $C=\log (2 \pi)-\zeta^{\prime}(2) / \zeta(2)-1$. 
The factor 4 only modifies the value of the constants.

Theorem 1.2. For every $\alpha>21 / 32$,

$$
\sum_{n \leq N} h(4 n) \log \varepsilon_{4 n}=\frac{4 \pi^{2}}{21 \zeta(3)} N^{3 / 2}-\frac{4}{\pi^{2}}\left(C^{\prime}+\log N\right) N+O\left(N^{\alpha}\right)
$$

where $C^{\prime}=\log (2 \pi)+8(\log 2) / 3-\zeta^{\prime}(2) / \zeta(2)-1$.

These theorems can be viewed as average results for real $L$-functions (cf. [Go-Ho], [Pe]).

Just to compare with the original claim in Art. 304 we state the last result in an equivalent form. Hereafter $\varepsilon$ indicates a positive arbitrarily small number (not always the same) and $O$ constants may depend on it.

COROLlaRY 1.3. The average order of $h(4 n) \log \varepsilon_{4 n}$ is

$$
f(n)=\frac{2 \pi^{2}}{7 \zeta(3)} n^{1 / 2}-\frac{4}{\pi^{2}} \log n-\frac{4}{\pi^{2}}\left(C^{\prime}+1\right)+O\left(n^{-11 / 32+\varepsilon}\right),
$$

i.e., $\sum_{n \leq N} h(4 n) \log \varepsilon_{4 n}=\sum_{n \leq N} f(n)$.

As in the case of negative discriminants, it seems difficult to prove a non-trivial $\Omega$-result for the error term in Theorems 1.1 and 1.2 , because it would require studying the correlation of the arithmetical function $N_{+}(n)$, introduced in the next section, with the Möbius function. On the other hand, the arguments of $[\mathrm{Ku}]$ or alternatively of $[\mathrm{Ts}]$ should yield an optimal $\Omega$ result for the average of $N_{+}(n)$. We thank Professor Kühleitner for providing us with his article.

2. Shintani's functional equations and summation formulas. In the 70's Sato and Shintani developed the notion of zeta functions associated to prehomogeneous vector spaces. In this setting Shintani [Sh] proved a vectorial functional equation for some zeta functions involving class numbers. His result and our summation formulas are better understood upon introducing the quantities

$$
N_{+}(n)=\sum_{k^{2} \mid n} h\left(n / k^{2}\right) \log \varepsilon_{n / k^{2}} \quad \text { and } \quad N_{-}(n)=\sum_{k^{2} \mid n} h\left(-n / k^{2}\right) \delta_{-n / k^{2}}^{-1}
$$

where $\delta_{-n}$ is half of the "number of units": $\delta_{-4}=2, \delta_{-3}=3$ and $\delta_{-n}=1$ otherwise. Note that $N_{-}(n)$ is, up to an additive constant appearing for $n=3 k^{2}$ or $n=4 k^{2}$, the class number of primitive and imprimitive forms of discriminant $-n$ (the Hurwitz class number). 
We can recover the class number easily by applying Möbius inversion.

Lemma 2.1. For $n \geq 1$,

$$
\begin{aligned}
h(n) \log \varepsilon_{n} & =\sum_{k^{2} \mid n} \mu(k) N_{+}\left(n / k^{2}\right), \\
h(4 n) \log \varepsilon_{4 n} & =\sum_{\substack{k^{2} \mid n \\
2 \nmid k}} \mu(k)\left(N_{+}\left(4 n / k^{2}\right)-N_{+}\left(n / k^{2}\right)\right) .
\end{aligned}
$$

Proof. The first formula follows easily. For the second, we have

$$
\begin{aligned}
h(4 n) \log \varepsilon_{4 n} & =\sum_{k^{2} \mid 4 n} \mu(k) N_{+}\left(4 n / k^{2}\right)=\sum_{2 \nmid k}+\sum_{2 \mid k} \\
& =\sum_{\substack{k^{2} \mid n \\
2 \nmid k}} \mu(k) N_{+}\left(4 n / k^{2}\right)+\sum_{k^{2} \mid n} \mu(2 k) N_{+}\left(n / k^{2}\right),
\end{aligned}
$$

and $\mu(2 k)=-\mu(k)$ for $k$ odd and vanishes for $k$ even.

Shintani introduced four Dirichlet series $\xi_{+}^{*}, \xi_{+}, \xi_{-}^{*}, \xi_{-}$and proved that they have meromorphic extensions and satisfy a functional equation relating the pair $\xi_{+}^{*}(3 / 2-s), \xi_{-}^{*}(3 / 2-s)$ to the pair $\xi_{+}(s), \xi_{-}(s)$. He used this functional equation (see Theorem 2.2) and a variant of Landau's Lemma to demonstrate Theorems 1.1 and 1.2 with $\alpha>3 / 4$.

Here we prefer to introduce two vectorial complex functions

$$
\vec{z}_{1}(s)=\left(\begin{array}{l}
\varrho_{1}(s) \\
\eta_{1}(s)
\end{array}\right) \quad \text { and } \quad \vec{z}_{2}(s)=\left(\begin{array}{l}
\varrho_{2}(s) \\
\eta_{2}(s)
\end{array}\right)
$$

where $\varrho_{j}$ and $\eta_{j}, j=1,2$, are determined by $\xi_{+}^{*}(s)=\varrho_{1}(2 s-1), \xi_{+}(s)=$ $\varrho_{2}(2 s-1), \xi_{-}^{*}(s)=\eta_{1}(2 s-1)$ and $\xi_{-}(s)=\eta_{2}(2 s-1)$. With this notation, in the region of absolute convergence $\Re s>2$, we have

$$
\begin{aligned}
& \eta_{1}(s)=\sum_{n=1}^{\infty} \frac{N_{-}(4 n)}{\sqrt{4 n}}(\sqrt{4 n})^{-s}, \quad \eta_{2}(s)=\sum_{n=1}^{\infty} \frac{N_{-}(n)}{\sqrt{n}}(\sqrt{n})^{-s}, \\
& \varrho_{1}(s)=\sum_{n=1}^{\infty} \frac{N_{+}(4 n)}{\sqrt{4 n}}(\sqrt{4 n})^{-s}+\sum \frac{\beta_{n}}{n^{s}} \\
& \varrho_{2}(s)=\sum_{n=1}^{\infty} \frac{N_{+}(n)}{\sqrt{n}}(\sqrt{n})^{-s}+\sum \frac{\alpha_{n}}{n^{s}}
\end{aligned}
$$

where

$$
\sum \frac{\alpha_{n}}{n^{s}}=\zeta(s)\left(\frac{\zeta^{\prime}(s+1)}{\zeta(s+1)}-\frac{\zeta^{\prime}(s)}{\zeta(s)}\right), \quad \sum \frac{\beta_{n}}{n^{s}}=\sum \frac{\alpha_{n}}{(2 n)^{s}}+\frac{\zeta(s) \log 2}{2^{s+1}-1} .
$$


We can write Theorem 2 of $[\mathrm{Sh}]$ as

THEOREM 2.2. With the previous notation the coordinate functions of

$$
\vec{z}_{1}(s)-\frac{1}{s-2}\left(\begin{array}{c}
\pi^{2} / 12 \\
\pi / 12
\end{array}\right)+\frac{1}{(s-1)^{2}}\left(\begin{array}{c}
1 / 2 \\
0
\end{array}\right)+\frac{1}{s-1}\left(\begin{array}{c}
\log (2 \pi) / 2 \\
1 / 4
\end{array}\right)
$$

and

$$
\vec{z}_{2}(s)-\frac{1}{s-2}\left(\begin{array}{c}
\pi^{2} / 6 \\
\pi / 6
\end{array}\right)+\frac{1}{(s-1)^{2}}\left(\begin{array}{l}
1 \\
0
\end{array}\right)+\frac{1}{s-1}\left(\begin{array}{c}
\log (2 \pi) \\
1 / 2
\end{array}\right)
$$

have entire continuations of order one. Moreover,

$$
\vec{z}_{2}(1-s)=(2 \pi)^{-s} \Gamma(s)\left(\mathcal{A}(s) \vec{z}_{1}(s)-\cos (\pi s / 2) \zeta(s) \vec{B}(s)\right)
$$

where

$\mathcal{A}(s)=2^{s+1}\left(\begin{array}{cc}\cos (\pi s / 2) & \pi \\ 0 & -\sin (\pi s / 2)\end{array}\right), \quad \vec{B}(s)=\left(\begin{array}{c}\psi(s / 2)-\psi((s+1) / 2) \\ \sec (\pi s / 2)\end{array}\right)$

with $\psi(s)=\Gamma^{\prime}(s) / \Gamma(s)$.

Proof. Use the change of variable $s \mapsto(2-s) / 2$, Theorem 2 of [Sh] and the duplication formula for $\Gamma$.

Now, we are ready to state and prove some summation formulas.

Proposition 2.3. Let $g \in C_{0}^{\infty}((0, \infty))$. Then

$$
\begin{aligned}
\sum_{n=1}^{\infty} \frac{N_{+}(n)}{\sqrt{n}} g(\sqrt{n})=\frac{\pi^{2}}{6} \int_{0}^{\infty} t g(t) d t-\int_{0}^{\infty} g(t) \log (2 \pi t) d t-\sum_{n=1}^{\infty} g(n) \log n \\
+\sum_{d=1}^{\infty} \frac{\Lambda(d)}{d} \sum_{n=1}^{\infty} g(d n)+2 \sum_{n=1}^{\infty} \frac{N_{+}(4 n)}{\sqrt{4 n}} \widetilde{g}(\sqrt{4 n})+2 \sum_{n=1}^{\infty} \beta_{n} \widetilde{g}(n) \\
+2 \pi \sum_{n=1}^{\infty} \frac{N_{-}(4 n)}{\sqrt{4 n}} \int_{0}^{\infty} g(t) e^{-\pi \sqrt{4 n} t} d t+2 \int_{0}^{1} \frac{1}{t(1+t)} \sum_{n=1}^{\infty} \widetilde{g}(2 n / t) d t
\end{aligned}
$$

where $\widetilde{g}$ indicates the Fourier cosine transform $\int_{0}^{\infty} g(t) \cos (\pi x t) d t$.

Proof. After inserting the notation, the summation formula is equivalent to

$$
\begin{aligned}
\sum_{n=1}^{\infty} b_{2 n}^{+} g(\sqrt{n})= & \frac{\pi^{2}}{6} \int_{0}^{\infty} t g(t) d t-\int_{0}^{\infty} g(t) \log (2 \pi t) d t+2 \sum_{n=1}^{\infty} b_{1 n}^{+} \widetilde{g}(\sqrt{n}) \\
& +2 \pi \sum_{n=1}^{\infty} b_{1 n}^{-} \int_{0}^{\infty} g(t) e^{-\pi \sqrt{n} t} d t+2 \int_{0}^{1} \frac{1}{t(1+t)} \sum_{n=1}^{\infty} \widetilde{g}(2 n / t) d t
\end{aligned}
$$

where $b_{i n}^{+}$and $b_{i n}^{-}$are determined by $\varrho_{i}(s)=\sum b_{i n}^{+}(\sqrt{n})^{-s}$ and $\eta_{i}(s)=$ $\sum b_{i n}^{-}(\sqrt{n})^{-s}$ 
By the Mellin inversion formula, we have

$$
\sum_{n=1}^{\infty} b_{2 n}^{+} g(\sqrt{n})=\frac{1}{2 \pi i} \int_{(\sigma)} G(s) \varrho_{2}(s) d s
$$

where $G(s)$ is the Mellin transform of $g, 2<\sigma<3$ and $(\sigma)$ indicates the vertical line $\Re s=\sigma$. Theorem 2.2 gives

$$
\begin{aligned}
& \varrho_{2}(1-s) \\
& =\pi^{-s} \Gamma(s)\left(2 \pi \eta_{1}(s)+2 \cos (\pi s / 2) \varrho_{1}(s)-2^{-s} \cos (\pi s / 2) B_{1}(s) \zeta(s)\right)
\end{aligned}
$$

where $B_{1}$ is the first coordinate of $\vec{B}$. This equation ensures (by convexity) that $\varrho_{2}(s)$ grows as a polynomial in vertical lines, so by the decay of $G(s)$ we can move the line of integration to $-2<\sigma^{\prime}<-1$. Thus from the residue theorem and the first part of Theorem 2.2 we get

$$
\sum_{n=1}^{\infty} b_{2 n}^{+} g(\sqrt{n})=\frac{1}{2 \pi i} \int_{\left(\sigma^{\prime}\right)} G(s) \varrho_{2}(s) d s+\frac{\pi^{2}}{6} G(2)-G^{\prime}(1)-\log (2 \pi) G(1) .
$$

Furthermore, by (3),

$$
\frac{1}{2 \pi i} \int_{\left(\sigma^{\prime}\right)} G(s) \varrho_{2}(s) d s=I_{1}+I_{2}+I_{3}
$$

where

$$
\begin{aligned}
& I_{1}=2 \pi \frac{1}{2 \pi i} \int_{(\sigma)} G(1-s) \pi^{-s} \Gamma(s) \eta_{1}(s) d s \\
& I_{2}=2 \frac{1}{2 \pi i} \int_{(\sigma)} G(1-s) \pi^{-s} \Gamma(s) \cos (\pi s / 2) \varrho_{1}(s) d s \\
& I_{3}=-\frac{1}{2 \pi i} \int_{(\sigma)} G(1-s)(2 \pi)^{-s} \Gamma(s) \cos (\pi s / 2) B_{1}(s) \zeta(s) d s .
\end{aligned}
$$

Using the series expansion for $\varrho_{1}(s)$ and moving the line of integration to $0<\sigma^{\prime \prime}<1$ gives

$$
I_{2}=2 \sum_{n=1}^{\infty} b_{1 n}^{+} \frac{1}{2 \pi i} \int_{\left(\sigma^{\prime \prime}\right)} G(1-s)(\pi \sqrt{n})^{-s} \Gamma(s) \cos (\pi s / 2) d s .
$$

In this range we can write $\Gamma(s) \cos (\pi s / 2)=\int_{0}^{\infty} t^{s-1} \cos t d t$ (see [Gr-Ry, 17.43.3]), hence

$$
I_{2}=2 \sum_{n=1}^{\infty} b_{1 n}^{+} \int_{0}^{\infty} \frac{1}{2 \pi i} \int_{\left(\sigma^{\prime \prime}\right)} G(1-s) t^{s-1} d s \cos (\pi \sqrt{n} t) d t
$$


and by the Mellin inversion formula it follows that

$$
I_{2}=2 \sum_{n=1}^{\infty} b_{1 n}^{+} \widetilde{g}(\sqrt{n}) \text {. }
$$

In the same way, we get

$$
I_{1}=2 \pi \sum_{n=1}^{\infty} b_{1 n}^{-} \int_{0}^{\infty} g(t) e^{-\pi t \sqrt{n}} d t .
$$

Finally, by the formula

$$
B_{1}(s)=-2 \int_{0}^{1} \frac{x^{s}}{1+x} \frac{d x}{x}
$$

in $\Re s>0$ (see [Gr-Ry, 8.371.1]), we can write

$$
I_{3}=2 \int_{0}^{1} \frac{1}{2 \pi i} \int_{(\sigma)} G(1-s)\left(2 \pi x^{-1}\right)^{-s} \Gamma(s) \cos (\pi s / 2) \zeta(s) d s \frac{1}{1+x} \frac{d x}{x} .
$$

Now, after expanding $\zeta$ into its Dirichlet series, we have

$$
I_{3}=2 \int_{0}^{1} \sum_{n=1}^{\infty} \widetilde{g}\left(2 n x^{-1}\right) \frac{1}{1+x} \frac{d x}{x}
$$

where we have proceeded as in $I_{2}$.

An analogous result holds for weighted sums of $N_{+}(4 n)$.

Proposition 2.4. Let $g \in C_{0}^{\infty}((0, \infty))$. Then

$$
\begin{aligned}
& \sum_{n=1}^{\infty} \frac{N_{+}(4 n)}{\sqrt{4 n}} g(\sqrt{4 n})=\frac{\pi^{2}}{12} \int_{0}^{\infty} t g(t) d t-\frac{1}{2} \int_{0}^{\infty} g(t) \log (2 \pi t) d t-\sum_{n=1}^{\infty} g(2 n) \log n \\
& \quad+\sum_{d=1}^{\infty} \frac{\Lambda(d)}{d} \sum_{n=1}^{\infty} g(2 d n)-\log 2 \sum_{k=1}^{\infty} 2^{-k} \sum_{n=1}^{\infty} g\left(2^{k} n\right)+\sum_{n=1}^{\infty} \frac{N_{+}(n)}{\sqrt{n}} \widetilde{g}(\sqrt{n}) \\
& \quad+\sum_{n=1}^{\infty} \alpha_{n} \widetilde{g}(n)+\pi \sum_{n=1}^{\infty} \frac{N_{-}(n)}{\sqrt{n}} \int_{0}^{\infty} g(t) e^{-\pi \sqrt{n} t} d t-\int_{0}^{1} \frac{1}{t(1+t)} \sum_{n=1}^{\infty} \widetilde{g}(n / t) d t
\end{aligned}
$$

Proof. We proceed as in the previous proposition, starting with the equation

$$
\varrho_{1}(1-s)=\pi^{-s} \Gamma(s)\left(\pi \eta_{2}(s)+\cos (\pi s / 2) \varrho_{2}(s)-\frac{1}{2} \cos (\pi s / 2) B_{1}(s) \zeta(s)\right),
$$

which we deduce by applying

$$
B_{1}(1-s)+B_{1}(s)=-2 \pi \csc (\pi s)
$$

in Theorem 2.2. 
3. An exponential sum. When applying Propositions 2.3 and 2.4 for a suitably chosen function $g$, we shall have to deal with oscillatory sums of the form

$$
\sum N_{+}(n) e(R \sqrt{n}) \text { and } \sum N_{+}(4 n) e(R \sqrt{n})
$$

where, as usual, $e(x)=e^{2 \pi i x}$.

A fundamental point is writing $N_{+}(n)$ as a sum over integral points. Thanks to the action of the modular group on the set of quadratic forms, this leads to a geometric discussion on the hyperbolic plane.

Consider Poincaré's model for the hyperbolic plane, given by the upper half plane $\mathbb{H}=\{x+i y: x \in \mathbb{R}, y>0\}$ endowed with the metric $d s^{2}=y^{-2}\left(d x^{2}+d y^{2}\right)$, and assign to each indefinite binary quadratic form $a x^{2}+b x y+c y^{2}$ with $a>0$ the geodesic $g_{a b c}$ in $\mathbb{H}$ given by (the Euclidean semicircle) $a\left(x^{2}+y^{2}\right)+b x+c=0, y>0$. Following [Si] we define $\mu(a, b, c)$ to be the hyperbolic length of the intersection of this geodesic with the standard fundamental domain:

$$
\mu(a, b, c)=\ell\left(g_{a b c} \cap \mathcal{F}\right) \quad \text { with } \quad \mathcal{F}=\left\{x+i y: x^{2}+y^{2}>1,|x|<1 / 2\right\} .
$$

The basic result that allowed Siegel to employ a lattice point approach for the average of $h(n) \log \varepsilon_{n}$ is the following

LEMMA 3.1. If $n \in \mathbb{Z}^{+}$is not a square then

$$
N_{+}(n)=\sum_{\substack{b^{2}-4 a c=n \\ a>0}} \mu(a, b, c) .
$$

Proof. By Lemma 2.1, this is a consequence of [Si, (44)] if we write $a=a^{\prime} k, b=b^{\prime} k, c=c^{\prime} k$ and sum over $k^{2} \mid D$ (note that $\mu(-a,-b,-c)=$ $\mu(a, b, c))$.

It is easy to see that there are only a finite number of non-vanishing terms in the sum. We shall quantify this situation:

LEMMA 3.2. Let $a x^{2}+b x y+c y^{2}, a>0$, have non-square positive discriminant $n$. Then $\mu(a, b, c) \neq 0$ if and only if $a+c<|b| / 2$. Moreover, if $\mu(a, b, c) \neq 0$ then $a \leq \sqrt{n / 3},|b| \leq 2 \sqrt{n / 3}$ and $a|c| \leq n / 4$.

Proof. See [Si, §6].

Our objective is to bound $\sum N_{+}(n) e(R \sqrt{n})$ as in [Ch-Iw2] but several differences arise: Firstly, the weight $\mu(a, b, c)$ involved does not appear in the case of negative discriminants (and the geometrical interpretation of $\mu$ shows that it can have large partial derivatives); secondly, $M \leq b^{2}-4 a c<2 M$ does not imply any a priori lower bound for $a, b$ and $c$ because $c$ need not be positive; finally, the contribution of square discriminants has to be excluded. 
Proposition 3.3. For $R>1 / 2$ and $1 \leq M<M^{\prime} \leq 2 M$,

$$
\sum_{M \leq n<M^{\prime}} N_{+}(n) e(R \sqrt{n}) \ll M^{5 / 4+\varepsilon}+M^{\varepsilon} L
$$

with

$$
L=\min \left(R^{3 / 8} M^{15 / 16}+R^{1 / 8} M^{17 / 16}, R^{7 / 24} M^{49 / 48}+R^{5 / 24} M^{53 / 48}\right),
$$

and a similar result holds when $N_{+}(n)$ is replaced by $N_{+}(4 n)$.

Proof. By Lemmas 3.2 and 3.1 we can write

$$
\sum_{M \leq n<M^{\prime}} N_{+}(n) e(R \sqrt{n})=\sum_{\substack{a+c<|b| / 2 \\ b^{2}-4 a c \neq \square}} \mu(a, b, c) E\left(b^{2}-4 a c\right)
$$

where

$$
E(n)= \begin{cases}e(R \sqrt{n}) & \text { if } M \leq n<M^{\prime} \\ 0 & \text { otherwise }\end{cases}
$$

Consider the set

$$
\mathcal{M}=\mathcal{M}_{1} \cup \mathcal{M}_{2} \cup \mathcal{M}_{3}
$$

where the disjoint sets $\mathcal{M}_{j}$ are defined by

$$
\begin{aligned}
& \mathcal{M}_{1}=\left\{(a, b, c) \in \mathbb{Z}^{3}:|a+c|<-b / 2, a>0, c \neq 0\right\}, \\
& \mathcal{M}_{2}=\left\{(a, b, c) \in \mathbb{Z}^{3}: a+c \leq b / 2 \leq-a-c, a>0, c \neq 0\right\}, \\
& \mathcal{M}_{3}=\left\{(a, b, c) \in \mathbb{Z}^{3}:|a+c|<b / 2, a>0, c \neq 0\right\} .
\end{aligned}
$$

The vertices $1 / 2+i \sqrt{3} / 2$ and $-1 / 2+i \sqrt{3} / 2$ of $\mathcal{F}$ both belong to the semicircle determined by $g_{a b c}$ if and only if $(a, b, c) \in \mathcal{M}_{2}$; in the same way, only the first vertex or only the second vertex belongs to this semicircle if and only if $(a, b, c) \in \mathcal{M}_{1}$ or $(a, b, c) \in \mathcal{M}_{3}$, respectively. Hence, $\mathcal{M}$ covers all the geometrical possibilities with $\mu(a, b, c) \neq 0$.

By [Si, (26)], on each $\mathcal{M}_{i}$ the function $\mu=\mu(a, b, c)$ is the logarithm of an algebraic function, moreover $\mu(a, b, c)=O\left(M^{\varepsilon}\right)$ when $M \leq b^{2}-4 a c<2 M$.

Note that $\mu(a, b, c)$ is also well defined as $\ell\left(g_{a b c} \cap \mathcal{F}\right)$ when $b^{2}-4 a c$ is a square (we shall impose later $c \neq 0$ ) and we still have $\mu(a, b, c)=O\left(M^{\varepsilon}\right)$. On the other hand, the quantity

$$
\#\left\{(a, b, c): b^{2}-4 a c=h^{2}, M \leq h^{2}<2 M, a+c<|b| / 2, a>0, c \neq 0\right\}
$$

is $O\left(M^{1+\varepsilon}\right)$ because the number of divisors of $b^{2}-h^{2}$ is $O\left(M^{\varepsilon}\right)$ and there are $O\left(M^{1+\varepsilon}\right)$ possibilities for the pair $b, h$ (a sharp estimate is possible following Art. 206 of [Ga]). Then from (4) we have, for some $i \in\{1,2,3\}$,

$$
\begin{aligned}
\sum_{M \leq n<M^{\prime}} N_{+}(n) e(R \sqrt{n}) & \ll M^{1+\varepsilon}+\sum_{(a, b, c) \in \mathcal{M}} \mu(a, b, c) E\left(b^{2}-4 a c\right) \\
& \ll M^{1+\varepsilon}+\sum_{(a, b, c) \in \mathcal{M}_{i}} \mu(a, b, c) E\left(b^{2}-4 a c\right) .
\end{aligned}
$$


Fix $a, c$ and consider $\mu(a, b, c)$ as a function of $b$. As $e^{\mu}$ is algebraic and non-constant in $\mathcal{M}_{i}$, it has a uniformly bounded number of maxima and minima. Hence given $a, c$ we can write $\left\{b:(a, b, c) \in \mathcal{M}_{i}\right\}$ as a finite union of intervals $I_{j}$ in which $\mu(a, \cdot, c)$ is monotonic. The bound $\mu(a, b, c) \ll M^{\varepsilon}$ and partial summation give

$$
\sum_{(a, b, c) \in \mathcal{M}_{i}} \mu(a, b, c) E\left(b^{2}-4 a c\right) \ll M^{\varepsilon} \sum_{a, c}\left|\sum_{b \in I_{j}^{\prime}} E\left(b^{2}-4 a c\right)\right| .
$$

The interval $I_{j}^{\prime} \subset I_{j}$ depends on $a, c$ and, of course, will be empty if $(a, b, c) \notin$ $\mathcal{M}_{i}$ for every $b$. Lemma 3.2 ensures $a|c| \leq M / 2$ and $|b| \leq 2 \sqrt{M}$; then Lemma 7.3 of $[\mathrm{Gr}-\mathrm{Ko}]$ applied on $[-2 \sqrt{M}, 2 \sqrt{M}]$ and the change of variable $n=4 a|c|$ give

$$
\sum_{a, c}\left|\sum_{b \in I_{j}^{\prime}} E\left(b^{2}-4 a c\right)\right| \ll M^{\varepsilon} \sum_{n \leq 2 M}\left|\sum_{|b| \leq 2 \sqrt{M}} e(\theta b) E\left(b^{2}-n\right)\right|
$$

for some $\theta \in \mathbb{R}$.

Now we follow the idea of Lemma 4.1 in [Ch-Iw2]. Let us divide the range of $b$ into $M^{\varepsilon}$ intervals of length $O\left(M^{1 / 2-\varepsilon}\right)$. If $J$ is one of these intervals, by Cauchy's inequality we get

$$
\begin{aligned}
\left(\sum_{n \leq 2 M} \mid \sum_{b \in J} e(\theta b) E\right. & \left.\left(b^{2}-n\right) \mid\right)^{2} \\
& \ll M\left(M^{3 / 2}+\sum_{\left|b_{1}\right|<\left|b_{2}\right|}\left|\sum_{n} E\left(b_{1}^{2}-n\right) \overline{E\left(b_{2}^{2}-n\right)}\right|\right) .
\end{aligned}
$$

Write $u=b_{1}^{2}-n$. Then the last double sum is

$$
\begin{aligned}
& \sum_{\left|b_{1}\right|<\left|b_{2}\right|} \mid \sum_{M \leq u \leq M^{\prime}+b_{1}^{2}-b_{2}^{2}} e(R(\sqrt{u}\left.\left.-\sqrt{u+b_{2}^{2}-b_{1}^{2}}\right)\right) \mid \\
& \ll M^{\varepsilon} \sum_{v \asymp D}\left|\sum_{u \asymp M} e(R(\sqrt{u}-\sqrt{u+v}))\right|
\end{aligned}
$$

for some $D=o(M)$, where we have employed the fact that the number of representations of $v$ as $b_{2}^{2}-b_{1}^{2}$ is $O\left(M^{\varepsilon}\right)$ and $b_{2}^{2}-b_{1}^{2}=o(M)$ because $|J|=o\left(M^{1 / 2}\right)$.

From all of these results, we finally obtain

$$
\begin{aligned}
\sum_{M \leq n<M^{\prime}} N_{+}(n) e(R \sqrt{n}) & \\
& \ll M^{5 / 4+\varepsilon}+M^{1 / 2+\varepsilon}\left(\sum_{v \asymp D}\left|\sum_{u \asymp M} e(R(\sqrt{u}-\sqrt{u+v}))\right|\right)^{1 / 2} .
\end{aligned}
$$

This was bounded in Lemma 3.1 of [Ch-Iw1], giving the desired result. 
The proof for $N_{+}(4 n)$ is similar; note that $4 \mid b^{2}-4 a c$ is equivalent to $2 \mid b$ and $2 \sum_{2 \mid b} f(b)=\sum_{b} f(b)+\sum_{b} e(b / 2) f(b)$, hence the phase $b / 2$ can be absorbed into $\theta b$ in (5).

4. Proof of the main results. Now we proceed as in [Ch-Iw2] and [Ch-Iw1] smoothing the summation and approximating, rather than bounding, the contribution of the last terms.

A new technical difficulty in the positive discriminants case is that we need more regularity than in the former cases to apply the summation formulas.

For $\Delta>0$, let $g: \mathbb{R} \rightarrow \mathbb{R}$ be given by

$$
g(x)= \begin{cases}\int_{0}^{x} \eta(u) d u & \text { if } x \leq 1 \\ x & \text { if } 1 \leq x \leq N^{1 / 2} \\ N^{1 / 2} \Delta^{-1}\left(N^{1 / 2}+\Delta-x\right) & \text { if } N^{1 / 2} \leq x \leq N^{1 / 2}+\Delta, \\ 0 & \text { if } x \geq N^{1 / 2}+\Delta,\end{cases}
$$

where $\eta \in C_{0}^{\infty}((1 / 2,1))$ with $\int_{0}^{1} \eta=1$. Note that $g \in C_{0}((0, \infty))$ and is piecewise differentiable.

Proposition 4.1. If $N^{-1 / 2}<\Delta \leq N^{-1 / 4}<1$, then

$$
\begin{aligned}
\sum_{n=1}^{\infty} \frac{N_{+}(n)}{\sqrt{n}} & g(\sqrt{n}) \\
= & \frac{\pi^{2}}{18} N^{3 / 2}+\frac{\pi^{2} N \Delta}{12}-\frac{N}{2} \log N+\left(1-\frac{\zeta^{\prime}(2)}{\zeta(2)}-\log (2 \pi)\right) \frac{N}{2} \\
& +O\left(N^{21 / 32+\varepsilon}+N^{1 / 2+\varepsilon} \Delta^{-1 / 2}+N^{11 / 16+\varepsilon} \Delta^{1 / 8}\right)
\end{aligned}
$$

and

$$
\begin{aligned}
\sum_{n=1}^{\infty} \frac{N_{+}(4 n)}{\sqrt{4 n}} & g(\sqrt{4 n}) \\
= & \frac{\pi^{2}}{36} N^{3 / 2}+\frac{\pi^{2} N \Delta}{24}+\left(1-\frac{\zeta^{\prime}(2)}{\zeta(2)}+\frac{\log 2}{3}-\log (2 \pi)\right) \frac{N}{4} \\
& -\frac{N}{4} \log N+O\left(N^{21 / 32+\varepsilon}+N^{1 / 2+\varepsilon} \Delta^{-1 / 2}+N^{11 / 16+\varepsilon} \Delta^{1 / 8}\right)
\end{aligned}
$$

REMARK. This proposition by itself allows us to improve the result of [Sh], because taking $\Delta=N^{-1 / 3}$ we conclude that the error term in the smoothed sum is $O\left(N^{2 / 3+\varepsilon}\right)$, and subtracting the same result after replacing $N^{1 / 2}$ by $N^{1 / 2}-\Delta$ we see that the contribution of the terms with $N^{1 / 2} \leq$ $\sqrt{n} \leq N^{1 / 2}+\Delta$ is absorbed by this error term. 
Proof. We prove the first formula and later indicate the changes necessary to prove the second.

A calculation shows that for $x>0$,

$$
\begin{aligned}
\widetilde{g}(x)= & \frac{\cos \left(\pi N^{1 / 2} x\right)-\cos (\pi x)-\phi(x)}{\pi^{2} x^{2}} \\
& +\frac{2 N^{1 / 2}}{\pi^{2} x^{2} \Delta} \sin \left(\frac{\pi}{2} \Delta x\right) \sin \left(\frac{\pi}{2}\left(2 N^{1 / 2}+\Delta\right) x\right)
\end{aligned}
$$

where $\phi(x)=\pi x \int_{0}^{\infty} \eta(t) \sin (\pi x t) d t$. Note that $\phi(x)=O\left(x^{-\alpha}\right)$ for every $\alpha>0$.

Let $\tau \in C_{0}^{\infty}((-1 / 2,1 / 2))$ be even with $\int \tau=1$, and $\tau_{m}(x)=m \tau(m x)$ for $m \in \mathbb{N}$. Define $g_{m}=g * \tau_{m}$. Then $g_{m} \in C_{0}^{\infty}((0, \infty))$ and $\widetilde{g}_{m}(x)=$ $\widetilde{g}(x) \widehat{\tau}(x / 2 m)$ converge uniformly to $g$ and $\widetilde{g}$. Moreover, by Proposition 3.3 we deduce that the sum involving $\sum N_{+}(n) \widetilde{g}(\sqrt{n}) / \sqrt{n}$ converges, and then by Abel's Lemma the sums $\sum_{n} N_{+}(n) \widetilde{g}_{m}(\sqrt{n}) / \sqrt{n}$ converge uniformly in $m$. This justifies the application of Proposition 2.3 for $g$.

In the assumed range of $\Delta$ we have

$$
\frac{\pi^{2}}{6} \int_{0}^{\infty} t g(t) d t=\frac{\pi^{2}}{18} N^{3 / 2}+\frac{\pi^{2} N \Delta}{12}+O(1)
$$

It is obvious that

$$
\int_{0}^{\infty} g(t) \log (2 \pi t) d t=\frac{N}{4} \log N+(2 \log (2 \pi)-1) \frac{N}{4}+O\left(N^{1 / 2+\varepsilon}\right)
$$

and by partial summation

$$
\begin{aligned}
\sum_{n=1}^{\infty} g(n) \log n-\sum_{d=1}^{\infty} \frac{\Lambda(d)}{d} \sum_{n=1}^{\infty} g(d n) & \\
= & \frac{N}{4} \log N-\frac{N}{4}+\frac{\zeta^{\prime}(2)}{2 \zeta(2)} N+O\left(N^{1 / 2+\varepsilon}\right) .
\end{aligned}
$$

The terms involving sums of $\widetilde{g}(2 n / t), \beta_{n}$ and $N_{-}(4 n)$ are negligible: their contributions can be proved to be $O\left(N^{1 / 2+\varepsilon}\right)$ using

$$
\widetilde{g}(x) \ll x^{-2}+N^{1 / 2} \min \left(x^{-1}, \Delta^{-1} x^{-2}\right),
$$

$\beta_{n} \ll \log n$ and $N_{-}(4 n)=O\left(n^{1 / 2+\varepsilon}\right)$.

By (6), the remaining sum $\sum N_{+}(4 n) \widetilde{g}(\sqrt{4 n}) / \sqrt{n}$ can be written, up to a multiplicative constant, as 


$$
\begin{aligned}
\sum_{n=1}^{\infty} \frac{N_{+}(4 n)}{n^{3 / 2}}(\cos (2 \pi \sqrt{N n})-\cos (2 \pi \sqrt{n})-\phi(\sqrt{4 n})) \\
\quad+2 \frac{N^{1 / 2}}{\Delta}\left(\sum_{n<N^{1 / 2}}+\sum_{N^{1 / 2} \leq n<\Delta^{-2}}+\sum_{n \geq \Delta^{-2}}\right) \frac{N_{+}(4 n)}{n^{3 / 2}} \sin (\pi \Delta \sqrt{n}) \\
\quad \times \sin \left(\pi\left(2 N^{1 / 2}+\Delta\right) \sqrt{n}\right) \\
=S_{0}+S_{1}+S_{2}+S_{3} .
\end{aligned}
$$

Firstly, the decay of $\phi$ and Proposition 3.3 prove that $S_{0} \ll \log N$. For $S_{1}$, note that $\sqrt{n} \Delta \ll 1$ and the factor $n^{-3 / 2} \sin (\pi \sqrt{n} \Delta)$ can be extracted by partial summation. Using Proposition 3.3 with the second value of the minimum gives

$$
S_{1} \ll\left(N^{5 / 8}+N^{7 / 48} N^{49 / 96}+N^{5 / 48} N^{53 / 96}\right) N^{\varepsilon} \ll N^{21 / 32+\varepsilon} .
$$

For $S_{2}$ we proceed in the same way but using this time the first value of the minimum to get

$$
\begin{aligned}
S_{2} & \ll N^{21 / 32+\varepsilon}+N^{1 / 2+\varepsilon} \Delta^{2}\left(\Delta^{-5 / 2}+N^{3 / 16} \Delta^{-15 / 8}+N^{1 / 16} \Delta^{-17 / 8}\right) \\
& \ll N^{21 / 32+\varepsilon}+N^{1 / 2+\varepsilon} \Delta^{-1 / 2}+N^{11 / 16+\varepsilon} \Delta^{1 / 8} .
\end{aligned}
$$

Finally, for $S_{3}$ we do not use partial summation, but use directly Proposition 3.3 as in $S_{2}$ to get the same bound.

(The first named author would like to take this opportunity to mention that in the last two inequalities on p. 427 of [Ch-Iw1], analogous to the bounds of $S_{2}$ and $S_{3}$, the terms $R^{5 / 16}$ and $R^{1 / 8} H^{7 / 8}$ should be added to the expression in parentheses. We thank Professor Kuba for pointing this out. These terms are negligible, once $H$ is chosen optimally, and do not affect the rest of the arguments.)

Collecting these results we have

$$
\sum_{n=1}^{\infty} \frac{N_{+}(4 n)}{n^{3 / 2}} \widetilde{g}(\sqrt{4 n}) \ll N^{21 / 32+\varepsilon}+N^{1 / 2+\varepsilon} \Delta^{-1 / 2}+N^{11 / 16+\varepsilon} \Delta^{1 / 8},
$$

which gives the desired result.

For the second formula of the statement, note that

$$
\sum_{n=1}^{\infty} g(2 n) \log n=\frac{N}{8} \log N-\frac{N}{8}-\frac{N}{4} \log 2+O\left(N^{1 / 2+\varepsilon}\right)
$$

and that the next two terms in Proposition 2.4 contribute

$$
\frac{N}{4} \sum_{d=1}^{\infty} \frac{\Lambda(d)}{d^{2}}-\log 2 \sum_{k=1}^{\infty} 2^{-2 k-1} N+O\left(N^{1 / 2+\varepsilon}\right)
$$

If we take this into account, the proof is entirely similar. 
Now we use a strong character sum estimate due to Heath-Brown (see [He]) to prove

Proposition 4.2. For $g, N$ and $\Delta$ as before,

$$
\begin{aligned}
\sum_{N^{1 / 2}<\sqrt{n}<N^{1 / 2}+\Delta} \frac{N_{+}(n)}{\sqrt{n}} g(\sqrt{n}) & =\frac{\pi^{2} N \Delta}{12}+O(E), \\
\sum_{N^{1 / 2}<\sqrt{4 n}<N^{1 / 2}+\Delta} \frac{N_{+}(4 n)}{\sqrt{4 n}} g(\sqrt{4 n}) & =\frac{\pi^{2} N \Delta}{24}+O(E),
\end{aligned}
$$

where $E=N^{11 / 12+\varepsilon} \Delta^{5 / 6}+N^{7 / 12+\varepsilon} \Delta^{-1 / 6}+N^{19 / 30+\varepsilon}$.

Proof. We have $h(n) \log \varepsilon_{n} \neq 0$ if and only if $n \in \mathcal{R}$, where $\mathcal{R}=\{n \in$ $\left.\mathbb{Z}^{+}: n \equiv 0,1(\bmod 4), n \neq \square\right\}$. Moreover in this case we have Dirichlet's class number formula [La]

$$
h(n) \log \varepsilon_{n}=\sqrt{n} L\left(1, \chi_{n}\right)
$$

where $\chi_{n}(m)=\left(\frac{n}{m}\right)$ is Kronecker's symbol. So we can write the left side of the first identity in the proposition as

$$
\begin{aligned}
\sum_{N^{1 / 2}<d \sqrt{a}<N^{1 / 2}+\Delta} & h(a) \log \varepsilon_{a} \frac{g(d \sqrt{a})}{d \sqrt{a}} \\
& =\sum_{d<N^{1 / 2}+\Delta} \frac{1}{d} \sum_{\substack{N d^{-2}<a<\left(N^{1 / 2}+\Delta\right)^{2} d^{-2} \\
a \in \mathcal{R}}} g(d \sqrt{a}) L\left(1, \chi_{a}\right)
\end{aligned}
$$

and by Abel's summation formula this is

$$
\frac{N^{1 / 2}}{2 \Delta} \sum_{d<N^{1 / 2}+\Delta} \frac{1}{d} \int_{0}^{2 \Delta N^{1 / 2}+\Delta^{2}} \frac{C\left(N d^{-2}, x d^{-2}\right)}{(N+x)^{1 / 2}} d x
$$

where

$$
C(x, K)=\sum_{\substack{x<n<x+K \\ n \in \mathcal{R}}} L\left(1, \chi_{n}\right) .
$$

But from $[\mathrm{He}]$ we have the estimate

$$
C(x, K)=\frac{\zeta(2)}{\zeta(3)} \frac{K}{2}+x^{\varepsilon} O\left(K^{5 / 6}+x^{2 / 15}+x^{1 / 6} \min \left(1, K^{-1 / 4}\right)\right)
$$

for any $0<K \ll x^{1 / 2}$. Now we substitute it in (7) and the first identity follows. For the second, the proof is similar. 
Corollary 4.3. For $N>1$,

$$
\begin{aligned}
\sum_{n \leq N} N_{+}(n)= & \frac{\pi^{2}}{18} N^{3 / 2}-\frac{N}{2} \log N+\left(1-\frac{\zeta^{\prime}(2)}{\zeta(2)}-\log (2 \pi)\right) \frac{N}{2} \\
& +O\left(N^{21 / 32+\varepsilon}\right), \\
\sum_{n \leq N} N_{+}(4 n)= & \frac{2 \pi^{2}}{9} N^{3 / 2}-N \log N+\left(1-\frac{\zeta^{\prime}(2)}{\zeta(2)}-\frac{5 \log 2}{3}-\log (2 \pi)\right) N \\
& +O\left(N^{21 / 32+\varepsilon}\right) .
\end{aligned}
$$

Proof. Subtract the last two results, use the fact that $g(\sqrt{n}) / \sqrt{n}=1$ for $1<n \leq N$ and choose $\Delta=N^{-5 / 16}$.

Proof of Theorem 1.1. By Lemma 2.1 we get the expression

$$
\sum_{n \leq N} h(n) \log \varepsilon_{n}=\sum_{k \leq \sqrt{N}} \mu(k) \sum_{n \leq N / k^{2}} N_{+}(n),
$$

and the result follows from Corollary 4.3. Note that $\sum k^{-2} \log k=-\zeta^{\prime}(2)$.

Proof of Theorem 1.2. Similarly, by the second part of Lemma 2.1,

$$
\sum_{n \leq N} h(4 n) \log \varepsilon_{4 n}=\sum_{\substack{k \leq \sqrt{N} \\ 2 \nmid k}} \mu(k)\left(\sum_{n \leq N / k^{2}} N_{+}(4 n)-\sum_{n \leq N / k^{2}} N_{+}(n)\right),
$$

and again the result is a consequence of Corollary 4.3, upon noting that

$\sum_{2 \nmid k} \frac{\mu(k)}{k^{3}}=\frac{8}{7 \zeta(3)}, \quad \sum_{2 \nmid k} \frac{\mu(k)}{k^{2}}=\frac{8}{\pi^{2}}, \quad \sum_{2 \nmid k} \mu(k) \frac{\log k}{k^{2}}=\left(\frac{\log 2}{3}+\frac{\zeta^{\prime}(2)}{\zeta(2)}\right) \frac{8}{\pi^{2}}$

(for the last equality, compute the derivative of $\left(\left(2^{-s}-1\right) \zeta(s)\right)^{-1}$ at $s=2$ ).

\section{References}

[Ch-Iw1] F. Chamizo and H. Iwaniec, On the sphere problem, Rev. Mat. Iberoamer. 11 (1995), 417-429.

[Ch-Iw2] - - - On the Gauss mean-value formula for class number, Nagoya Math. J. 151 (1998), 199-208.

[Ga] C. F. Gauss, Disquisitiones Arithmeticae, Springer, New York, 1986.

[Go-Ho] D. Goldfeld and J. Hoffstein, Eisenstein series of 1/2-integral weight and the mean value of real Dirichlet L-series, Invent. Math. 80 (1985), 185-208.

[Gr-Ko] S. W. Graham and G. Kolesnik, Van der Corput's Method of Exponential Sums, London Math. Soc. Lecture Note Ser. 126, Cambridge Univ. Press, Cambridge, 1991.

[Gr-Ry] I. S. Gradshteyn and I. M. Ryzhik, Tables of Integrals, Series, and Products, 5th ed., Academic Press, Boston, MA, 1994.

[He] D. R. Heath-Brown, Lattice points in the sphere, in: Number Theory in Progress, Vol. 2 (Zakopane-Kościelisko, 1997), de Gruyter, Berlin, 1999, 883-892. 
[Ku] M. Kühleitner, On the class number of binary quadratic forms: an omega estimate for the error term, Math. Pannon. 13 (2002), 63-78.

[La] E. Landau, Elementary Number Theory, 2nd ed., Chelsea, New York, 1958.

[Li] R. Lipschitz, Über die asymptotischen Gesetzen von gewissen Gattungen zahlentheoretischer Funktionen, Monatsber. Königl. Akad. Wiss. Berlin 1865, 174-185.

[Pe] M. Peter, Mean values of Dirichlet L-series, Math. Ann. 318 (2000), 67-84.

[Sa1] P. Sarnak, Class numbers of indefinite binary quadratic forms, J. Number Theory 15 (1982), 229-247; Corrigenda, ibid. 16 (1983), 284.

[Sa2] -, Class numbers of indefinite binary quadratic forms. II, ibid. 21 (1985), 333-346.

[Sh] T. Shintani, On zeta-functions associated with the vector space of quadratic forms, J. Fac. Sci. Univ. Tokyo Sect. I A Math. 22 (1975), 25-65.

[Si] C. L. Siegel, The average measure of quadratic forms with given determinant and signature, Ann. of Math. (2) 45 (1944), 667-685.

[Ts] K.-M. Tsang, Counting lattice points in the sphere, Bull. London Math. Soc. 32 (2000), 679-688.

Departamento de Matemáticas

Universidad Autónoma de Madrid

28049 Madrid, Spain

E-mail: fernando.chamizo@uam.es

adrian.ubis@uam.es

Received on 13.6.2005

and in revised form on 28.10.2005 